\title{
Iodine-mediated oxidation of resveratrol. An electroanalytical study using platinum and glassy carbon electrodes
}

Beatriz Gómez-Monedero a , Rebeca Jiménez-Pérez ${ }^{a}$, María Isabel González-Sánchez ${ }^{\mathrm{a}}$, Loreno Almagro $^{\mathrm{b}}$, Edelmira Valero ${ }^{\mathrm{a} *}$ and Richard G. Compton ${ }^{\mathrm{c}}$

aDepartment of Physical Chemistry, Higher Technical School of Industrial Engineering, University of Castilla-La Mancha, Campus Universitario s/n, 02071, Albacete, Spain.

${ }^{b}$ Department of Plant Biology, Faculty of Biology, University of Murcia, Campus de Espinardo, 30100, Murcia, Spain.

'Department of Chemistry, Physical and Theoretical Chemistry Laboratory, University of Oxford, South Parks Road, Oxford OX1 3QZ, United Kingdom.

*Address correspondence to Edelmira Valero: Edelmira.valero@uclm.es. 


\section{ABSTRACT}

Resveratrol is a phenolic compound that shows important biological antioxidant activities. In this paper, the electrochemical oxidation of iodide in the presence of resveratrol was investigated using both platinum and glassy carbon electrodes. The experimental results showed a diffusion controlled process for the oxidation of iodide to iodine, followed by the chemical reaction of iodine with resveratrol. The possible applicability of iodine-mediated reaction in the quantitative analysis of resveratrol was studied, obtaining sensitivities of $0.98 \pm 0.03$ and $4.22 \pm 0.20 \mu \mathrm{AMM}^{-1} \mathrm{~cm}^{-2}$ using platinum and glassy carbon electrodes, respectively. The resveratrol content in a sample obtained from the extracellular medium of elicited suspension-cultured cells of Vitis vinifera was evaluated and compared with data obtained by HPLC analysis, with good correlations. The signal of other compounds that may be present in the samples or they are precursors of the biosynthesis of trans-resveratrol (L-ascorbic, glycine, saccharose, tyrosine, cinnamic acid and $p$-coumaric acid) was also evaluated, which shows lower interferences when using glassy carbon electrodes.

\section{KEYWORDS}

Cyclic voltammetry; glassy carbon electrode; iodide/iodine redox couple; platinum electrode; resveratrol. 


\section{INTRODUCTION}

trans-Resveratrol (3,5,4'-trihydroxy-trans-stilbene, trans-R) (Fig. 1) is a naturally occurring phytoalexin produced by a limited number of plants as a response to stress or injury, fungal attack or UV exposure [1]. This compound is a phenol derivative which has been found to potentially present antioxidant and antimutagen properties, to mediate anti-inflammatory effects or to inhibit hydroperoxidase functions [2]. This makes resveratrol a promising compound for applications as dietary supplement, functional food ingredient, cosmetics ingredient and even as a therapeutic agent [3]. Due to the high value of this compound, great effort has been made to produce it in high amounts. Some studies have demonstrated that the enrichment of Vitis vinifera cell cultures with cyclodextrins and methyljasmonate considerably enhanced the production of trans-R [4, 5]. Besides the search of new methods to produce high trans-R quantities, it is also very important to understand its reactivity and to develop fast and reliable analytical methods for its determination.

Given the great importance of this antioxidant compound, several analytical methods for its quantification have been developed such as liquid chromatography (which is the most frequently used) [6], gas chromatography [7] and capillary electrophoresis [8]. These methods provide excellent results, although they have some limitations such as being time-consuming, their high priced equipment and the laborious technical handling. In this context, electrochemical measurements for the quantification of trans-R can be a good alternative due to their advantages such as simplicity, cheapness and quickness. Some electrochemical sensors have been already reported for the quantification of transR. The electrochemical determination of this antioxidant has been carried out by cyclic voltammetry, differential pulse and square-wave voltammetry using a glassy carbon electrode [9]. Furthermore, electrodes based on indium tin oxide [10], multi-walled 
carbon nanotubes-modified glassy carbon [11], cyclodextrin modified carbon paste electrode [12] and biosensors based on peroxidase [13] have been published. In this paper, a new electroanalytical method is proposed as an alternative, using sodium iodide as an electrochemical mediator. Over existing sensors, this method is very simple, easy to use and avoids modification of conventional electrodes with expensive materials such as nanomaterials or biological compounds.

The electrochemical oxidation of iodide to iodine under aqueous conditions has been used as a redox mediator in the analytical measurement of several compounds such as biological thiols [14, 15], phenols [16] and antioxidant capacity [17]. The electrogenerated iodine is able to oxidize reduced compounds such as phenol, $o$-cresol, glutathione, cysteine, homocysteine, ascorbate, NADH and gallic acid, then the iodine turns back to iodide, which is again electrochemically oxidized resulting in an increase of the measurable current by virtue of the catalytic cycle.

The aim of this work was to perform an electrochemical study to investigate the iodine-mediated oxidation of resveratrol using both platinum and glassy carbon (GC) electrodes. In accordance with the electrochemical results, we have explored possible applications of this system for the analytical determination of trans-R obtained from elicited suspension-cultured cells of Vitis vinifera cv. Monastrell.

\section{EXPERIMENTAL}

\subsection{Reagents}

L-ascorbic acid, cinnamic acid, p-coumaric acid, ethanol, glycine, trans-R, saccharose, sodium iodide, sodium perchlorate and tyrosine were purchased from SigmaAldrich (Madrid, Spain). Perchloric acid was obtained from Merck (Madrid, Spain) and sulfuric acid from Panreac (Barcelona, Spain) All of them were purchased at their highest 
available purity and were used as received. All solutions were freshly prepared every day with deionized water (resistivity of no less than $18.2 \mathrm{M} \Omega \cdot \mathrm{cm}$ at $25^{\circ} \mathrm{C}$ ) (Millipore, Watford, UK).

Due to the low solubility of resveratrol in aqueous solutions, stock solutions of this compound were prepared daily in absolute ethanol and stored under darkness at $4{ }^{\circ} \mathrm{C}$. To perform the electrochemical measurements the appropriate volume of the stock solution was diluted to the desired concentration in $0.2 \mathrm{M} \mathrm{NaClO}_{4}$, adjusting the final concentration of ethanol to $15 \%$.

\subsection{Plant material and elicitor treatments}

Grapevine suspension-cultured cells derived from Vitis vinifera $\mathrm{cv}$. Monastrell calli were obtained and maintained as previously described $[5,18]$. In order to obtain trans-R, 15 day old suspension-cultured cells of $V$. vinifera were grown in the presence of cyclodextrins (Wacker Chemie, Spain) and methyljasmonate (Duchefa, Spain). For that, $20 \mathrm{~g}$ of fresh cells were added to $250 \mathrm{~mL}$ flasks which contained $100 \mathrm{~mL}$ of culture medium [5] supplemented with $100 \mu \mathrm{M}$ methyljasmonate and $50 \mathrm{mM}$ cyclodextrins. These suspension-cultured cells of $V$. vinifera were maintained at $25^{\circ} \mathrm{C}$ in the dark in a rotary shaker $(110 \mathrm{rpm})$ for $144 \mathrm{~h}$. Once the elicitation was finished, grapevine cells were separated from the culture medium by filtration, and the extracellular medium was used for trans-R quantification.

\subsection{Extraction of trans-resveratrol}

trans-R was extracted from $100 \mathrm{~mL}$ of elicited cell culture media by phase partitioning with ethyl acetate. The organic phase was collected and evaporated at $35^{\circ} \mathrm{C}$ in vacuum. This procedure was performed three times in order to extract all the trans-R present in the culture medium. The residue was redissolved in $5 \mathrm{~mL}$ methanol and 
evaporated on a glass petri dish until obtaining a precipitate. After that, the precipitate was triturated to achieve a powder enriched in trans-R.

\subsection{Electrochemical measurements}

Electrochemical experiments were performed using a potentiostat AUTOLAB PGSTAT128N (Eco Chemie B.V., The Netherlands) controlled by the sofware NOVA. Electrochemical measurements were carried out using a three-electrode cell $(10 \mathrm{ml})$ maintained at $25 \pm 0.5^{\circ} \mathrm{C}$ with a circulating water bath. The working electrodes (WE) used were: a glassy carbon electrode (GCE) of $3 \mathrm{~mm}$ diameter $(\mathrm{CH}$ Instruments), or a $\mathrm{Pt}$ electrode of $3 \mathrm{~mm}$ diameter (Methrom); the reference electrode (RE) was $\mathrm{Ag} / \mathrm{AgCl}$ (saturated $\mathrm{KCl})(\mathrm{CH}$ Instruments) and the counter electrode $(\mathrm{CE})$ was a $\mathrm{Pt}$ sheet (Methrom).

Renewal of GC electrode surface was achieved by immersing the electrode into a concentrated chromic mixture solution for $5 \mathrm{~min}$ and then further rinsing with deionized water. Renewal of Pt surface was achieved by polishing with three successive alumina slurry pastes $(1,0.3$ and $0.05 \mu \mathrm{m})$ and the residual abrasive particles were ultrasonically removed in deionized water.

The electroactive area of Pt electrode was calculated from the underpotential deposition portion of its $\mathrm{H}_{2} \mathrm{SO}_{4}$ profile, after considering the double layer capacity [19]. The electroactive area of GC electrode was calculated from the Randles-Sevcik equation using $10 \mathrm{mM}$ hexaammineruthenium (III) chloride at different scan rates [20].

\subsection{HPLC analysis}

HPLC equipment was an Agilent 1200 series instrument (Agilent Technologies, Madrid, Spain) equipped with an on-line vacuum degasser, quaternary pump, autosampler, temperature-controlled sample tray, thermostatted column compartment and 
diode array detector (DAD). The HPLC column was a reversed-phase C18 Eclipse XDB (4.6 $\mathrm{x} 150 \mathrm{~mm}, 5 \mu \mathrm{m}$ particle size). The HPLC grade solvents used were water as solvent A and acetonitrile as solvent B, previously filtered through a $0.22 \mu \mathrm{m}$ filter and degassed by sonication in a Selecta Ultrasons water bath. After the HPLC column was equilibrated, a mixture of $90 \%$ solvent $\mathrm{A}$ and $10 \%$ solvent $\mathrm{B}$ was maintained for $7 \mathrm{~min}$, followed by a linear gradient to obtain $85 \%$ solvent $\mathrm{B}$ at $18 \mathrm{~min}$. Then, $85 \%$ of solvent $\mathrm{B}$ was maintained until $30 \mathrm{~min}$, with a linear gradient to obtain $10 \%$ at $33 \mathrm{~min}$ [21].

Samples were filtered through a $0.45 \mu \mathrm{m}$ filter prior to injection. Elution conditions were as follows: injection volume, $20 \mu \mathrm{L}$; flow rate, $1.0 \mathrm{~mL} / \mathrm{min}$; oven temperature, $40{ }^{\circ} \mathrm{C}$. The elution was monitored at $306 \mathrm{~nm}$. Calibration straight lines were performed using standard trans-R by duplicate injection. An Agilent ChemStation B.03.02 revision was used to integrate peak areas. 


\section{RESULTS AND DISCUSSION}

This work reports the electrochemical oxidation of iodide to iodine and the reaction of the electro-generated iodine with trans-R at both platinum and glassy carbon electrodes together with its possible applicability in the analysis of trans- $\mathrm{R}$ in in vitro bioproduced real samples.

\subsection{Electrochemical performance}

Figure 2 shows voltammograms of $3 \mathrm{mM} \mathrm{NaI}$ in the absence and presence of trans-R both in platinum and glassy carbon electrodes. A quasi-reversible redox wave for the iodide/iodine redox couple at a mid-point potential of $\sim 0.47 \mathrm{~V}(v s \mathrm{Ag} / \mathrm{AgCl})$ was observed in both cases (solid black lines), which is similar to data previously reported $[14,15,17,22]$.

The voltammetric peaks found correspond to the one-electron oxidation of the iodide ion, although the oxidation to triodide ion formation could be also possible [23]. As regards the Pt electrode (Figure 2A), in the presence of trans-R (dashed black line), the voltammogram showed an increase in the forward peak and a decrease in the back peak suggestive of catalytic behavior as seen for the iodine mediated oxidation of As(III) on boron-doped diamond electrodes [24] and the iodine mediated electrochemical oxidation of thiols in platinum [15]. In the absence of the mediator NaI (solid grey line), redox peaks of trans-R were not observed (in the micromolar range of concentration).

Solutions of trans-R were prepared in water containing $15 \%$ of ethanol due to the low solubility of this compound in aqueous solutions. The electrochemical adsorption and oxidation of ethanol on platinum-based electrodes has been widely studied $[25,26]$. Figure 3 shows the cyclic voltammetry profile for the electro-oxidation of ethanol (15\%) in a solution of $0.2 \mathrm{M} \mathrm{NaClO}_{4}$, where an intense peak at $0.5 \mathrm{~V}$ (vs $\left.\mathrm{Ag} / \mathrm{AgCl}\right)$ was observed, 
which is in the range of potentials for NaI oxidation. However, in the presence of iodine and/or trans-R, this peak was not observed (Figure 2A), suggesting that these compounds compete with ethanol, and that iodine and trans- $\mathrm{R}$ present much more affinity for the $\mathrm{Pt}$ surface than ethanol. The strong adsorption of iodine on $\mathrm{Pt}$ is well known [27, 28]. For this reason, it was concluded that ethanol does not interfere in the measurements performed in this work.

When the GC electrode was used (Figure 2B), the electrochemical oxidation/reduction voltammogram of the system iodide/iodine in the absence of trans-R was similar to the case of Pt (Figure 2B, solid black line). However, the electrochemical behaviour of the system considerably changed in the presence of trans-R (Figure 2B, dashed black line). In this case, the voltammogram exhibits a higher increase in the anodic current and a decrease of the cathodic current in the reverse scan. In addition, a shift of the oxidation potential to more positive potentials occurred (from 0.5 to $0.65 \mathrm{~V} v s$ $\mathrm{Ag} / \mathrm{AgCl}$ ). This displacement to more positive potentials could be due to the adsorption of trans-R or possibly iodine on the surface of the electrode, which inhibits to a certain extent the performance of the electrodic process. Similar behavior was observed in the electrochemical study of iodide in the presence of barbituric acid [29], and phenol and $o$ cresol [16], where the deposition of the product on the surface of the electrode was considered as the reason for the anodic shift.

The above assumption was corroborated by performing successive cycles of $3 \mathrm{mM}$ iodide/iodine in the presence of $60 \mu \mathrm{M}$ of resveratrol without cleaning between measurements (Figure 1S(A), Supporting Information). The signal decreased and the anodic peak was displaced towards more positive potentials. However, when the GC electrode was cleaned with chromic-sulfuric mixture, the cyclic voltammetries were identical (Figure 1S(B), Supporting Information). 


\subsection{Scan rate study}

Figures $4 \mathrm{~A}$ and $\mathrm{B}$ show the cyclic voltammetries of $3 \mathrm{mM} \mathrm{NaI}$ in the absence (solid lines) and presence of trans-R (dotted lines) at different scan rates using both electrodes. Once again, a shift in the oxidation peak of iodide towards more positive potentials was observed for the case of GC electrode, in contrast to the Pt one. Furthermore, the change in current intensity was also quite higher using GC electrode. In both cases, the scan rate study revealed linear double logarithmic plots of anodic peak of iodide $v s$ scan rate with a slope close to 0.5 both in the absence and presence of trans-R (Figures 4C and D), consistent with the theoretical slope for diffusion controlled processes [30]. These results suggest that all the trans- $\mathrm{R}$ that can diffusionally reach the electrode is turned over on the timescale of the voltammetry. Iodide diffusion coefficient (in $0.2 \mathrm{M} \mathrm{NaClO}_{4}$ with $15 \% \mathrm{EtOH}$ ) at $\mathrm{pH} 4$ was calculated in the absence of trans- $R$ from the slope of the plot I $v s v^{1 / 2}$ by the Randles-Sevcik equation. The value obtained using Pt and GC electrodes resulted to be $(1.2 \pm 0.1) \times 10^{-5} \mathrm{~cm}^{2} \mathrm{~s}^{-1}$, which compares well with literature values $[24,31]$.

The variation of the ratio $\mathrm{Ip} / \mathrm{Ir} / \mathrm{Ip}_{\mathrm{cr}}$ (anodic peak current intensity/cathodic peak current intensity) of the system iodide/iodine in the presence of trans-R as a function of scan rate obtained from Figure 4, for both electrodes, is shown in Figure 5A. In all cases, $\mathrm{I} \mathrm{p}_{\mathrm{ar}} / \mathrm{Ip} \mathrm{p}_{\mathrm{cr}}$ ratios were higher than unity, which might be attributed to complex coupled chemical reactions involving iodine, resveratrol and phenoxy radicals of resveratrol since iodine is a known reagent for iodination of phenols [32] as well as the involvement of adsorbed species. Furthermore, the fact that the ratio $\mathrm{Ip}_{\mathrm{a}} / \mathrm{Ip}_{\mathrm{c}}$ is more pronounced at the slowest scan rates, is consistent with a catalytic reaction [33]. Importantly the current function $\mathrm{Ip}_{\mathrm{a}} / \mathrm{v}^{1 / 2}$ decreased with scan rate in both cases, suggesting also that there are chemical steps coupled to the oxidation of iodide (Figure 5B). Therefore, the diagnostic 
obtained from Figure 5 points out that the oxidation of iodide in the presence of trans-R undergoes 'follow up' chemistry [30]. This behavior is different to the reaction of electrogenerated iodine with thiols $[14,15]$ and other antioxidants [17], in which a simple EC' mechanism operates.

\subsection{Selectivity}

In order to assess the application of the electrooxidation of iodide to measure resveratrol, real samples of this compound bioproduced by suspension-cultured cells of $V$. vinifera $\mathrm{cv}$. Monastrell elicited with methyljasmonate in the presence of cyclodextrins were tested [5]. Previously, the effect of some important chemicals that can coexist with trans-R in this kind of samples were analysed (Figure 6). The compounds checked were L-ascorbic acid, $p$-coumaric acid, glycine, saccharose, tyrosine and trans-cinnamic acid. Using the Pt electrode, a slightly increase in the current signal was observed in the presence of these compounds, at the same potential than trans-R (Figure 6A). However, when these compounds coexist with resveratrol in the same sample, the voltammetric signal coincides with the resveratrol signal in the absence of them (Figure 6B). Different behavior was observed with the GC electrode (Figures 6C and D). The compounds tested did not shift the oxidation potential of iodide while a large displacement of the potential was again obtained in the presence of resveratrol, both in the presence or the absence of the rest of compounds. In addition, at this range of concentrations, the increment in the current signal of the iodide/iodine couple after addition of these compounds with GC electrode was negligible (Figure 6C). When these compounds and trans-R were mixed in solution, voltammograms obtained coincided with that obtained in the presence of only trans-R (Figure 6D). Therefore, we infer that these compounds do not interfere in the analytical method when they coexist with trans-R in the same sample both in Pt and GC electrodes. This behavior is not inconsistent with a competition of these compounds in 
their reaction with iodine, with trans-R the compound which simply reacts the faster. Taking into account the above results, GC electrode is preferable for the quantitative analysis of trans-R, due to its better sensitivity and the displacement of the peak potential when resveratrol is present.

\subsection{Analytical parameters}

Calibration plots of resveratrol were performed in order to demonstrate the possible applicability of these electrochemical systems to the analytical measurement of resveratrol in real samples. Figure 7A shows the cyclic voltammograms of the couple iodide/iodine with different concentrations of resveratrol using the Pt electrode. This electrode gave a linear range for resveratrol between 15 and $120 \mu \mathrm{M}$ (inset, Figure 7A), with a calibration equation of $\mathrm{j}-\mathrm{j}_{0}\left(\mu \mathrm{A} \cdot \mathrm{cm}^{-2}\right)=0.84+0.98 \cdot \mathrm{C}_{\text {resveratrol }}(\mu \mathrm{M})$. The limit of detection and sensitivity obtained were $5.5 \mu \mathrm{M}$ and $0.98 \pm 0.04 \mu \mathrm{A}_{\mu} \mathrm{M}^{-1} \mathrm{~cm}^{-2}$, respectively. As regards the GC electrode, this electrode showed a linear range between 5 and $75 \mu \mathrm{M}$ resveratrol (inset, Figure $7 \mathrm{~B})$, with a calibration equation of $\mathrm{j}-\mathrm{j}_{0}\left(\mu \mathrm{A} \cdot \mathrm{cm}^{-2}\right)$ $=-3.20+4.22 * \mathrm{C}_{\text {resveratrol }}(\mu \mathrm{M})$. For this case, the sensitivity obtained was $4.22 \pm 0.2$ $\mu \mathrm{A} \mu \mathrm{M}^{-1} \mathrm{~cm}^{-2}$ and the limit of detection $2.3 \mu \mathrm{M}$. Therefore, the sensitivity obtained with the GC electrode was more than 4 times higher than that obtained when using the $\mathrm{Pt}$ electrode, and the LOD was near 2.4 times lower. Therefore, GC electrode shows better analytical parameters than the Pt one towards the analysis of resveratrol with NaI. The higher sensitivity obtained with the GC electrode to the analysis of trans-R probably reflects the preconcentration of this compound, due to adsorption on the electrode, prior to reaction with electrogenerated iodine.

\subsection{Real samples measurement}


In accordance with the above, the quantification of trans-R in real samples of this compound was evaluated using the GC electrode by using iodide solution and measuring its oxidative peak by voltammetry. In particular, we measured the trans-R concentration in powders enriched in trans-R obtained from elicited suspension-cultured cells of $V$. vinifera $\mathrm{cv}$. Monastrell [5]. The concentration obtained with the electrochemical method was $24.1 \pm 2.4 \%$, while a content of $24.9 \pm 1.0 \%$ was obtained by HPLC (standard method). These results show that the trans-R content matched very well to the values obtained by the standard method, with a recovery of $96.8 \%$.

\section{CONCLUSIONS}

The use of the iodide/iodine redox couple as a mediator was investigated for the electrochemical analysis of resveratrol using platinum and glassy carbon electrodes. In both cases and in the presence of trans- $R$, voltammograms showed an increase in the forward peak and a decrease in the back peak, suggestive of catalytic behavior. However, a shift of the anodic potential to more positive values took place in the case of the GC electrode. This displacement was attributed to the adsorption of trans- $R$ or iodine on the surface of GC electrode. The scan rate study showed an electrochemical diffusion controlled oxidation of iodide followed by chemical reactions between the electrogenerated iodine and trans-R (EC mechanism) both for platinum and GC electrodes. In addition, no interference effects were obtained when trans-R was mixed with L-ascorbic acid, p-coumaric acid, glycine, saccharose, tyrosine and trans-cinnamic acid. Better analytical parameters were attained when using GC electrode than those obtained using the platinum electrode (sensitivity more than 4 times higher and LOD 2.4 times lower), probably due to a higher preconcentration of trans-R on the surface of GC electrode by adsorption. Therefore, this electrode is analytically preferable and it was

chosen for the development of a method for quantification of resveratrol. The 
methodology herein proposed exhibited a sensitivity of $4.22 \pm 0.2 \mu \mathrm{A}_{\mu} \mathrm{M}^{-1} \mathrm{~cm}^{-2}$ and a limit of detection of $2.3 \mu \mathrm{M}$. Moreover, trans-R was successfully analysed in a bioproduced real sample by the developed methodology as a proof-of-concept and validated independently with a standard method (HPLC). 


\section{FUNDING SOURCE}

This work was funded by the Spanish Ministry of Economy and Competitiveness

(MINECO, http://www.mineco.gob.es/portal/site/mineco/idi), Project No. BFU201675609-P (AEI/FEDER, EU), and by the Junta de Comunidades de Castilla-La Mancha (Spain), Project No. SBPLY/17/180501/000276/2 (cofunded with FEDER funds, EU). BGM is a post-doctoral research fellow of the Youth Employment Initiative (JCCM, Spain, cofunded with ESF funds, EU). The funders had no role in study design, data collection and analysis, decision to publish, or preparation of the manuscript. 


\section{REFERENCES}

[1] L. Fremont, Minireview - Biological effects of resveratrol, Life Sciences, 66 (2000) 663-673.

[2] M.S. Jang, E.N. Cai, G.O. Udeani, K.V. Slowing, C.F. Thomas, C.W.W. Beecher, H.H.S. Fong, N.R. Farnsworth, A.D. Kinghorn, R.G. Mehta, R.C. Moon, J.M. Pezzuto, Cancer chemopreventive activity of resveratrol, a natural product derived from grapes, Science, 275 (1997) 218-220.

[3] M. Chu, L. Almagro, B. Chen, L. Burgos, M.A. Pedreno, Recent trends and comprehensive appraisal for the biotechnological production of trans-resveratrol and its derivatives, Phytochemistry Reviews, 17 (2018) 491-508.

[4] M. Chu, M.A. Pedreno, N. Alburquerque, L. Faize, L. Burgos, L. Almagro, A new strategy to enhance the biosynthesis of trans-resveratrol by overexpressing stilbene synthase gene in elicited Vitis vinifera cell cultures, Plant Physiology and Biochemistry, 113 (2017) 141-148.

[5] S. Belchí-Navarro, L. Almagro, D. Lijavetzky, R. Bru, M.A. Pedreno, Enhanced extracellular production of trans-resveratrol in Vitis vinifera suspension cultured cells by using cyclodextrins and methyljasmonate, Plant Cell Reports, 31 (2012) 81-89.

[6] I. Kolouchova-Hanzlikova, K. Melzoch, V. Filip, J. Smidrkal, Rapid method for resveratrol determination by HPLC with electrochemical and UV detections in wines, Food Chemistry, 87 (2004) 151-158.

[7] P. Vinas, N. Campillo, N. Martínez-Castillo, M. Hernández-Córdoba, Solid-phase microextraction on-fiber derivatization for the analysis of some polyphenols in wine and grapes using gas chromatography-mass spectrometry, Journal of Chromatography A, 1216 (2009) 12791284.

[8] X.L. Gu, Q.Y. Chub, M. O'Dwyer, M. Zeece, Analysis of resveratrol in wine by capillary electrophoresis, Journal of Chromatography A, 881 (2000) 471-481.

[9] O. Corduneanu, P. Janeiro, A.M.O. Brett, On the electrochemical oxidation of resveratrol, Electroanalysis, 18 (2006) 757-762.

[10] H.Y. Xiang, W.G. Li, Electrochemical Sensor for trans-Resveratrol Determination Based on Indium Tin Oxide Electrode Modified with Molecularly Imprinted Self-Assembled Films, Electroanalysis, 21 (2009) 1207-1210.

[11] W.S. Huang, S.J. Luo, D.Z. Zhou, S.H. Zhang, K.B. Wu, Electrochemical Determination of Resveratrol Using Multi-Walled Carbon Nanotubes-Modified Glassy Carbon Electrode, Nanoscience and Nanotechnology Letters, 5 (2013) 367-371.

[12] B. Pekec, A. Oberreiter, S. Hauser, K. Kalcher, A. Ortner, Electrochemical Sensor Based on a Cyclodextrin Modified Carbon Paste Electrode for Trans-Resveratrol Analysis, International Journal of Electrochemical Science, 7 (2012) 4089-4098.

[13] A.M. Granero, H. Fernández, E. Agostini, M.A. Zon, An amperometric biosensor for transresveratrol determination in aqueous solutions by means of carbon paste electrodes modified with peroxidase basic isoenzymes from Brassica napus, Electroanalysis, 20 (2008) 858-864.

[14] E. Valero-Ruiz, M.I. González-Sánchez, C. Batchelor-McAuley, R.G. Compton, Halogen mediated voltammetric oxidation of biological thiols and disulfides, Analyst, 141 (2016) 144-149.

[15] M.I. González-Sánchez, E. Valero, R.G. Compton, lodine mediated electrochemical detection of thiols in plant extracts using platinum screen-printed electrodes, Sensors and Actuators BChemical, 236 (2016) 1-7.

[16] L. Fatouhi, M. Ganjavi, D. Nematollahi, Electrochemical study of iodide in the presence of phenol and o-cresol: Application to the catalytic determination of phenol and o-cresol, Sensors, 4 (2004) 170-180. 
[17] M.I. González-Sánchez, J. Agrisuelas, E. Valero, R. G. Compton, Measurement of total antioxidant capacity by electrogenerated iodine at disposable screen printed electrodes, Electroanalysis, 2017.

[18] S. Belchí-Navarro, L. Almagro, A.B. Sabater-Jara, F. Fernández-Perez, R. Bru, M.A. Pedreno, Induction of trans-resveratrol and extracellular pathogenesis-related proteins in elicited suspension cultured cells of Vitis vinifera cv Monastrell, Journal of Plant Physiology, 170 (2013) 258-264.

[19] S. Trasatti, O.A. Petrii, Real surface-area measurements in electrochemistry, Pure and Applied Chemistry, 63 (1991) 711-734.

[20] Y.J. Wang, J.G. Limon-Petersen, R.G. Compton, Measurement of the diffusion coefficients of $\mathrm{Ru}\left(\mathrm{NH}_{3}\right)_{6}{ }^{3+}$ and $\mathrm{Ru}\left(\mathrm{NH}_{3}\right)_{6}{ }^{2+}$ in aqueous solution using microelectrode double potential step chronoamperometry, Journal of Electroanalytical Chemistry, 652 (2011) 13-17.

[21] P. Jeandet, A.C. Breuil, M. Adrian, L.A. Weston, S. Debord, P. Meunier, G. Maume, R. Bessis, HPLC analysis of grapevine phytoalexins coupling photodiode array detection and fluorometry, Analytical Chemistry, 69 (1997) 5172-5177.

[22] R.P. Akkermans, F.L. Qiu, S.L. Roberts, M.F. Suarez, R.G. Compton, Laser-activated voltammetry. Mechanism of aqueous iodide oxidation at platinum electrodes: Theory and experiment, Journal of Physical Chemistry B, 103 (1999) 8319-8327.

[23] C.L. Bentley, A.M. Bond, A.F. Hollenkamp, P.J. Mahon, J. Zhang, Voltammetric Determination of the lodide/lodine Formal Potential and Triiodide Stability Constant in Conventional and Ionic Liquid Media, Journal of Physical Chemistry C, 119 (2015) 22392-22403.

[24] G. Hignett, J.D. Wadhawan, N.S. Lawrence, D.Q. Hung, C. Prado, F. Marken, R.G. Compton, Electrochemical detection of $\mathrm{As}(\mathrm{III})$ via iodine electrogenerated at platinum, gold, diamond or carbon-based electrodes, Electroanalysis, 16 (2004) 897-903.

[25] R.G. Freitas, M.C. Santos, R.T.S. Oliveira, L.O.S. Bulhoes, E.C. Pereira, Methanol and ethanol electroxidation using Pt electrodes prepared by the polymeric precursor method, Journal of Power Sources, 158 (2006) 164-168.

[26] O. Guillén-Villafuerte, G. García, M. Carmen Arévalo, J. Luis Rodríguez, E. Pastor, New insights on the electrochemical oxidation of ethanol on carbon-supported Pt electrode by a novel electrochemical mass spectrometry configuration, Electrochemistry Communications, 63 (2016) 48-51.

[27] D.C. Johnson, Study of adsorption and desorption of iodine and iodide at platinum electrodes in $1 \mathrm{M}$ sulfuric acid, Journal of the Electrochemical Society, 119 (1972) 331-339.

[28] Z.X. Shu, S. Bruckenstein, lodine adsorption studies at platinum, Journal of Electroanalytical Chemistry, 317 (1991) 263-277.

[29] D. Nematollahi, M. Hesari, Electrochemical study of iodide in the presence of barbituric acid: application of the catalytic determination of barbituric acid, Journal of Analytical Chemistry, 56 (2001) 1109-1112.

[30] A.J. Bard, L.R. Faulkner, Electrochemical Methods: Fundamentals and Applications, Second Edition ed.2004.

[31] A.L. Beilby, A.L. Crittenden, Non-aditive polarographic waves in the anodic oxidation of iodide, Journal of Physical Chemistry, 64 (1960) 177-179.

[32] K. Omura, Oxidation of phenols with iodine in alkaline methanol, Journal of Organic Chemistry, 49 (1984) 3046-3050.

[33] C.E. Banks, R.G. Compton, Understanding voltammetry, $3^{\text {rd }}$ edition, World Scientific Press, Singapore, 2018. 


\section{FIGURE AND SCHEME CAPTIONS}

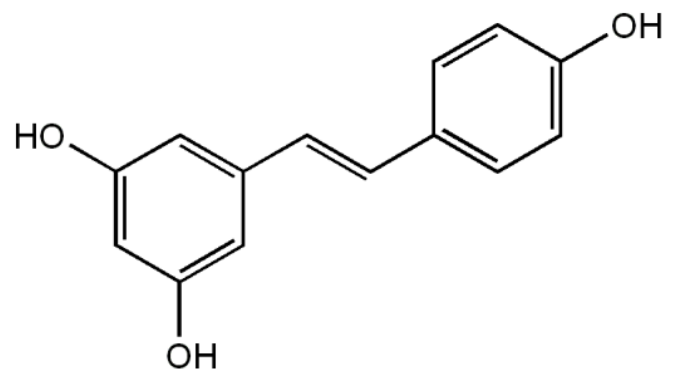

Figure 1. Chemical structure of trans-R. 

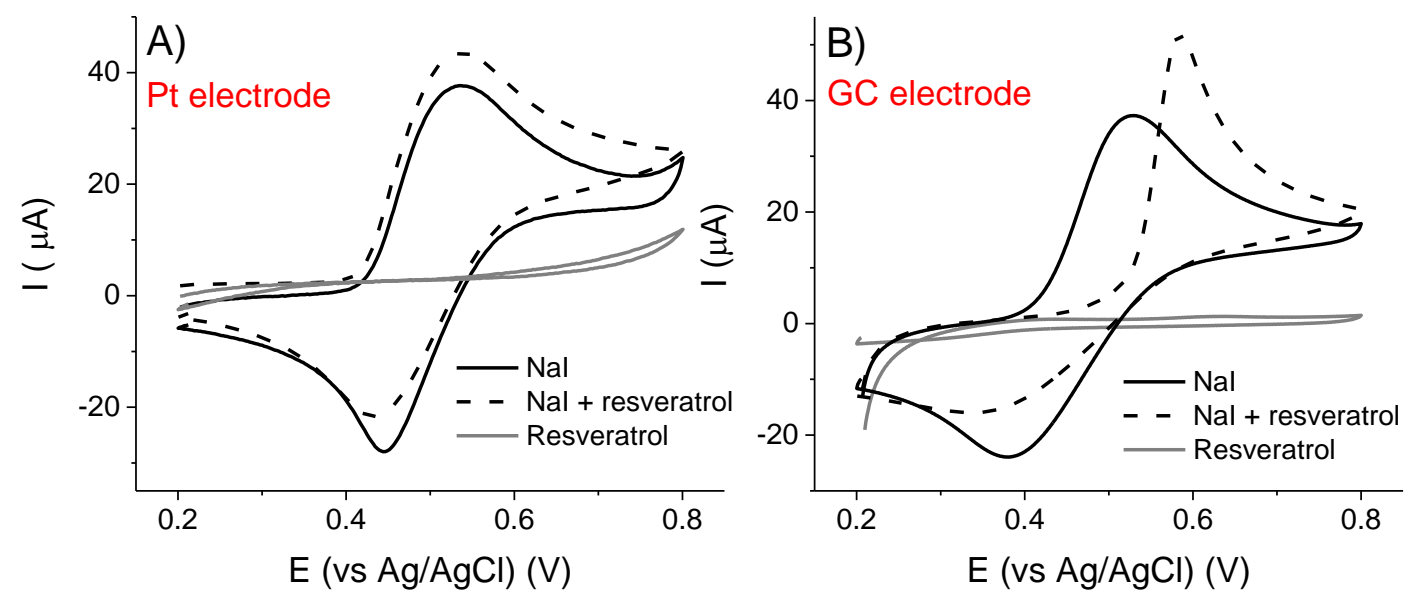

Figure 2. The voltammetric oxidation ( $\mathrm{scan}$ rate $=0.05 \mathrm{Vs}^{-1}$ ) of $3 \mathrm{mM} \mathrm{NaI}$ in the absence (solid black line) and presence of trans-R (dashed black line) using Pt (A) and glassy carbon (GC) electrodes (B) electrodes. The voltammetric response of trans-R in the absence of $\mathrm{NaI}$ is depicted by the grey line. The concentration of trans-R was $120 \mu \mathrm{M}$ in A) and $60 \mu \mathrm{M}$ in B). All solutions were prepared in $0.2 \mathrm{M} \mathrm{NaClO}_{4}$ with $15 \% \mathrm{EtOH}(\mathrm{pH}$ $=4)$. 


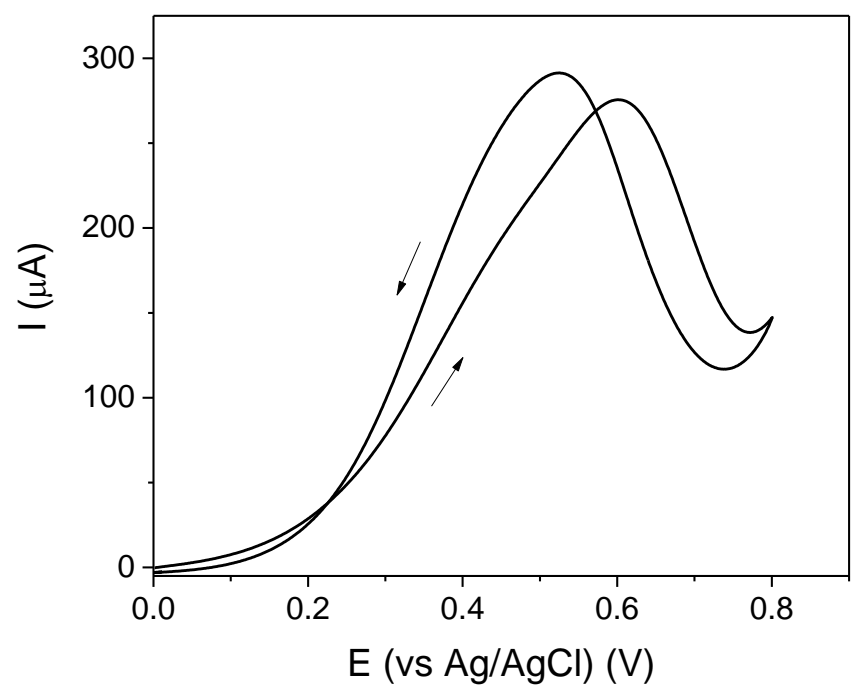

Figure 3. Linear sweep voltammetry for the electrooxidation of ethanol $(15 \%)$ in a solution of $0.2 \mathrm{M} \mathrm{NaClO}_{4}$ using a Pt electrode. 

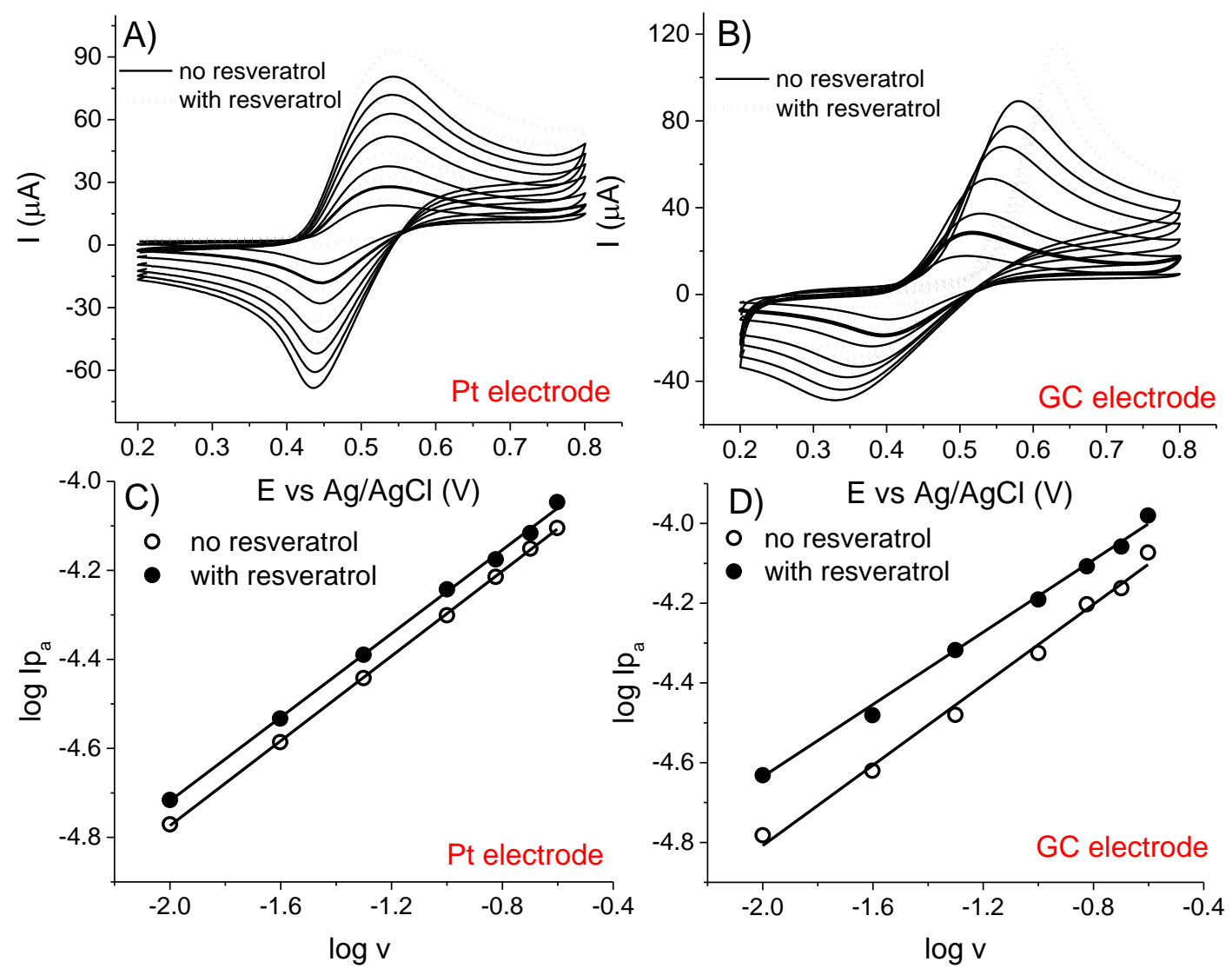

Figure 4. Cyclic voltammetries of $3 \mathrm{mM} \mathrm{NaI}$ in the absence (solid lines) and presence of trans-R (dotted lines) at different scan rates: 10, 25, 50, 100, 150, 200 and $250 \mathrm{mVs}^{-1}$ using Pt (A) and GC (B) electrodes. The concentration of trans-R was $120 \mu \mathrm{M}$ in A) and $60 \mu \mathrm{M}$ in B). (C) Plot of $\log \mathrm{Ip}_{\mathrm{a}} v s \log \mathrm{v}$ were calculated from Figure A). (D) Plot of $\log$ $\mathrm{Ip}_{\mathrm{a}} v s \log \mathrm{v}$ were calculated from Figure B). The $\mathrm{pH}$ of all solutions was 4 . 

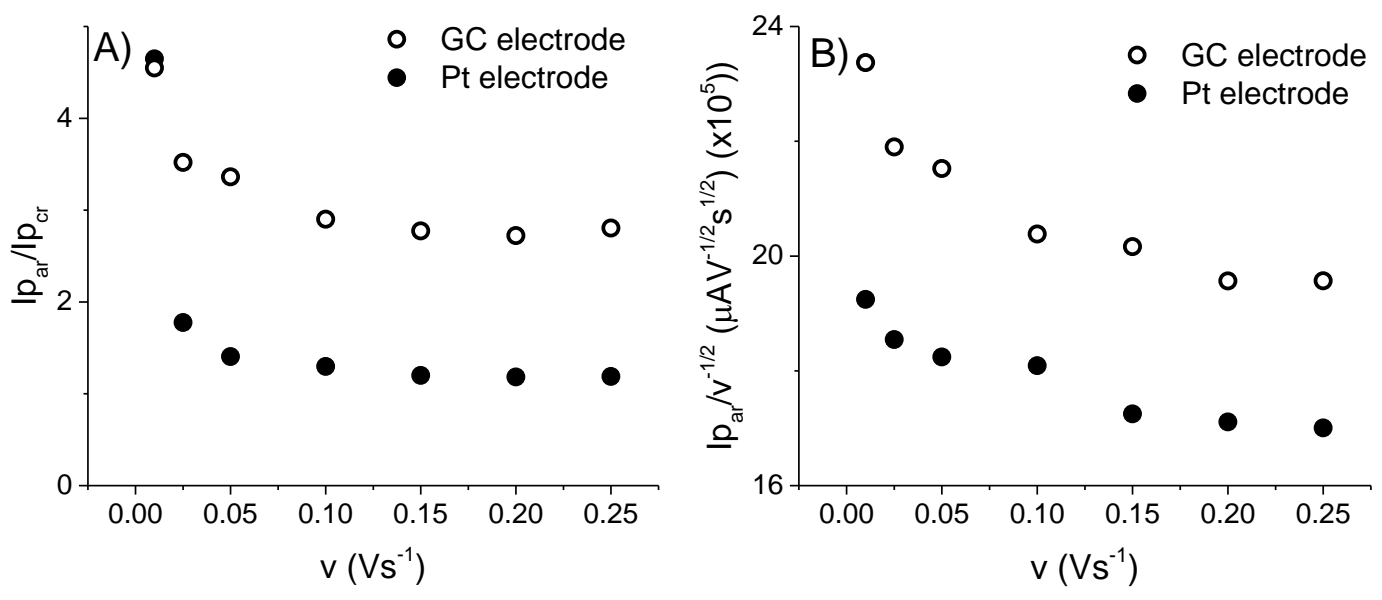

Figure 5. A) Variation of $I \mathrm{p}_{\mathrm{ar}} / \mathrm{I} \mathrm{p}_{\mathrm{cr}}$ for the oxidation and reduction peaks of the system iodide/iodine in the presence of trans-R as a function of scan rate using Pt (filled black circles) and GC (empty black circles) electrodes. B) Variation of the current function $\mathrm{Ip} / \mathrm{p}^{1 / 2}$ as a function of scan rate using the Pt electrode (filled black circles) and GC electrode (empty black circles). Data were obtained from Figure 3. 

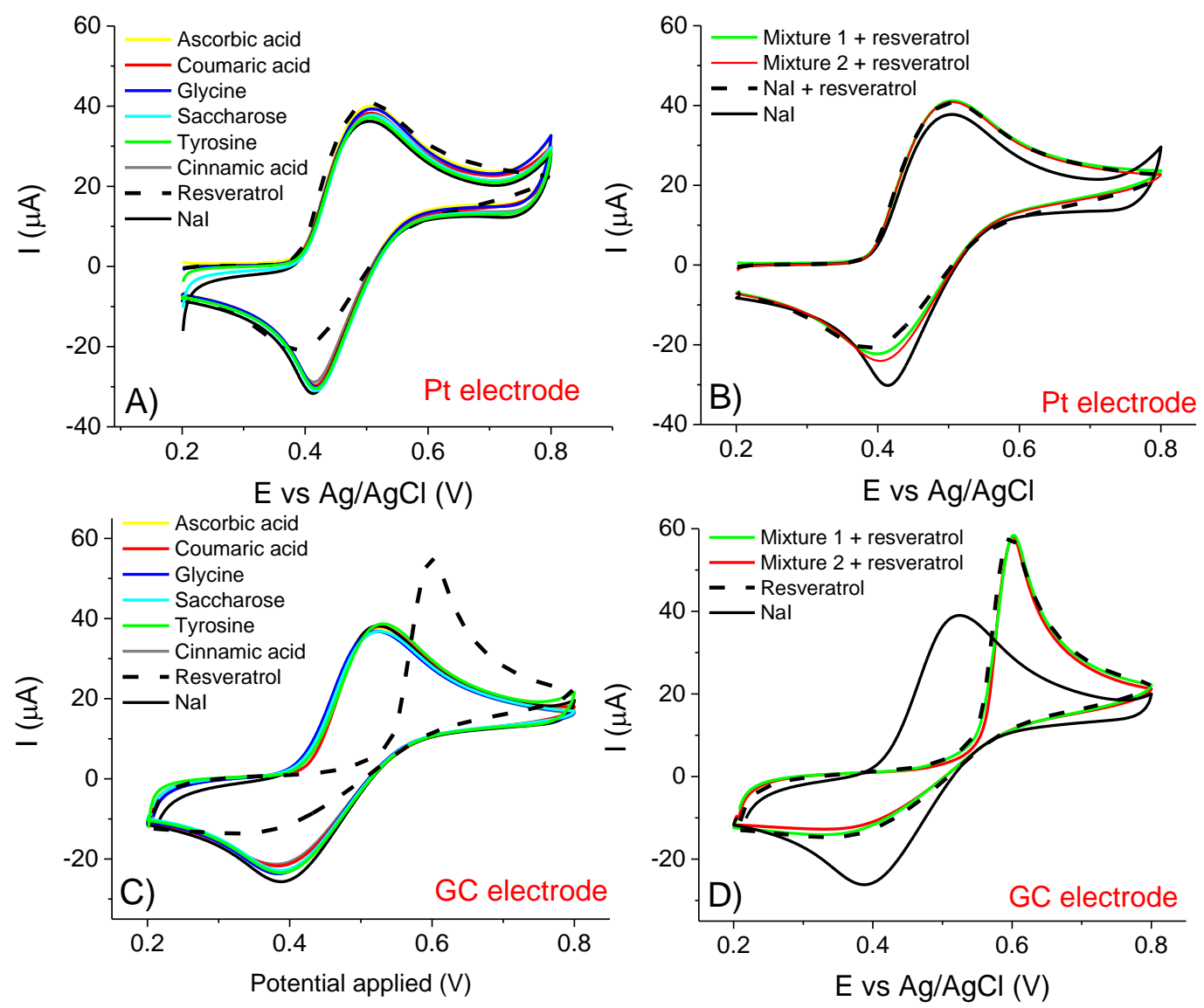

Figure 6. Cyclic voltammetries of $3 \mathrm{mM}$ iodide in the absence (solid black lines) and in the presence of trans- $\mathrm{R}$ (dashed black lines) and/or different compounds and mixtures of compounds that can be present in the samples using Pt ((A) and (B)) and GC electrodes ((C) and (D)). The experimental conditions were: $120 \mu \mathrm{M}$ of each compound when using Pt electrode and $60 \mu \mathrm{M}$ when using GC electrode. Mixture 1 contains L-ascorbic acid, cinnamic acid, $p$-coumaric acid and resveratrol. Mixture 2 contains glycine, tyrosine, saccharose and resveratrol. The scan rate was $50 \mathrm{mVs}^{-1}$ and the $\mathrm{pH} 4$. For greater clarity, samples containing $\mathrm{NaI}$ and resveratrol have been plotted with dashed lines. 

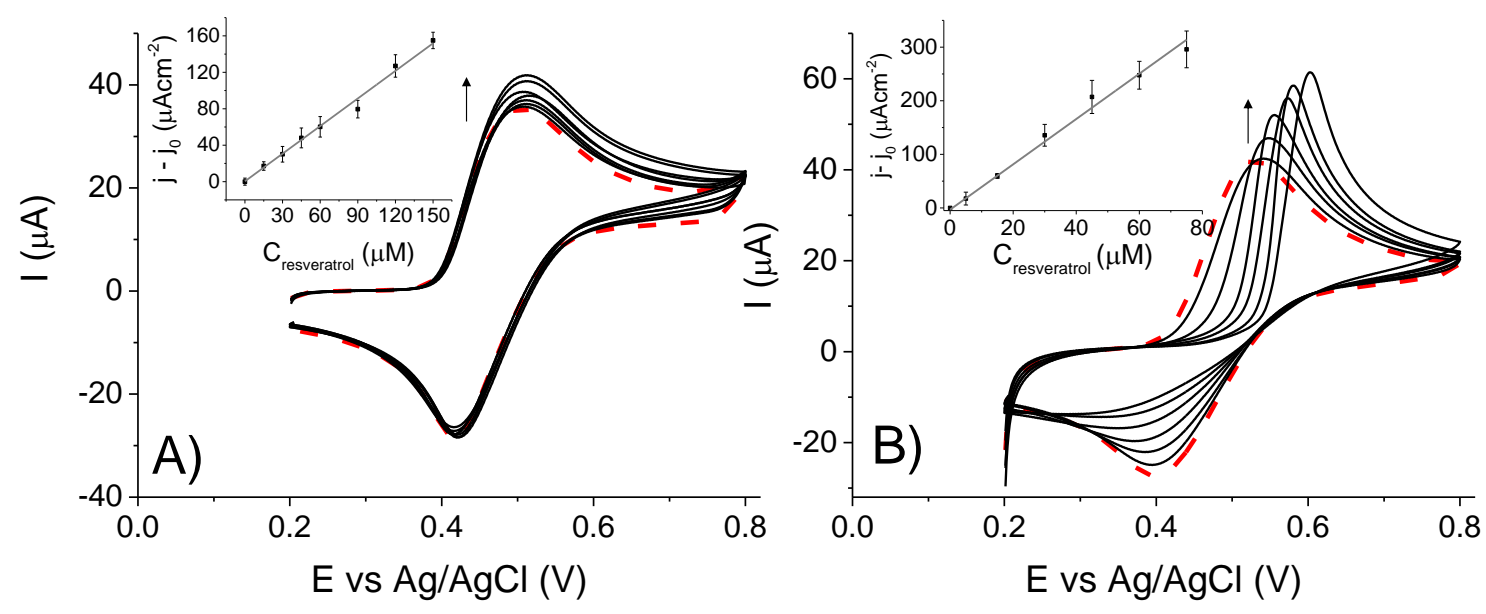

Figure 7. Voltammetric response of $\mathrm{NaI}$ in the absence (dashed red line) and presence of different increasing resveratrol concentrations (solid black lines). A) Pt electrode; experimental conditions: $3 \mathrm{mM} \mathrm{NaI}$ and $0,15,30,45,60,90,120$ and $150 \mu \mathrm{M}$ of resveratrol. Inset: Current densities of the anodic peak vs resveratrol concentration. B) GC electrode; experimental conditions: $3 \mathrm{mM} \mathrm{NaI}$ and $0,5,15,30,45,60$ and $75 \mu \mathrm{M}$ of resveratrol. Inset as indicated in A). Error bars were calculated as the standard deviation of three different measurements. The scan rate was $50 \mathrm{mVs}^{-1}$ and the $\mathrm{pH}$ was 4 . 